\title{
Thoughts on Trumpet Teaching of Music Education Major in Normal
}

\section{Universities}

\author{
Yan Jiyuan
}

School of Music, Shaanxi Normal University

Keywords: Trumpet; Education and teaching; Playing skills

\begin{abstract}
The music school is mainly set up to cultivate more professional talents for the future music circle, so the major music colleges must have a set of suitable teaching methods, which both cultivate the child's professional abilities, but also make children trained in a relaxed state. This paper mainly introduces the following aspects. Firstly, it gives a detailed introduction of the trumpet, mainly starting from the historical origins and basic characteristics of trumpet. Secondly, it introduces the performance skill training of trumpet from the perspective of to introduce, first of the trumpet were introduced in detail, mainly from the small the starting, followed by the trumpet breathing practice modes, long tone practice modes, mastery of rhythm and speed, ear listening training, music understanding and training as well as the cooperation of the overall team. It is hoped that this paper will provide a direction for the educational model in the academy and train more outstanding talents for the country in the future.
\end{abstract}

\section{Introduction of the Trumpet}

The history of trumpet is relatively long among all the instruments; it is a traditional instrument. And after the study, we have calculated the trumpet should have had the history of nearly four thousand years. It represents a kind of historical profoundness. The tones expressed by the trumpet are very bright and strong, enjoying an important status in the major instruments. In the long history, the trumpet has not always been enduring. Due to the fatal shortcomings of the trumpet in its design at the beginning, it also suffered from the hardship of nearly disappearing in the halfway. Later, through continuous transformation and improvement, it was ultimately retained. At present, it is an indispensable instrument element in various music.

I believe that most people have heard the sounds of the trumpet, which are very loud and very unique. As long as it is heard, it will be identified. Because of the characteristics of this sound, a lot of composers like this instrument very much and like its expressions with distinctive features. They hold that it can perfectly express the original creative ideas of composers. In addition, the trumpet's range is also very broad, especially the mid-range and treble of the trumpet are very expressive, so it is applied by many bands. The combination of trumpet and drum can play the very exciting music, so it has also been applied by the army bands. Of course, the trumpet's bass area is also very beautiful, with a mysterious beauty.

\section{Training of Trumpet's Playing Skills}

It is well known that trumpet playing is mainly done by the player's mouth, so there is a great demand for the athlete's vital capacity and breath. If they want to express the song perfectly through the trumpet, firstly, they should have a good performance method and a set of suitable breathing 
modes. At this stage, chest and abdomen mixed breathing method is used by many players, and is currently recognized as the most convenient and universal one, which can make performers play in an easy way to the fullest. It can also make the performers turn its breath amount to the maximum and flexibly control the playing breath. They can freely control both the high and low sound area. Such breath mode is the key to the perfect expression of the song.

In order to freely express the essence of the song in the performance, the players need to completely master this way of breathing, so they need to do some necessary exercises: first, relax the body and stand straight and the distance between the legs should be the same with that between shoulders. Then, put hands down and stretch downwards and meanwhile breathe deeply. They should have the sense of fullness in the waist and abdomen and slowly breathe the abdominal breath. Then this practice is over. Do multiple training with five practices as a group, which can help the player to effectively grasp this way of breathing.

\section{Method of Prolonged Sound}

In trumpet practice, the most important thing is the training of the prolonged sound. The performance of the prolonged sound has great demands for both performers and instrument itself. The performance training of the prolonged sound is helpful for students to cultivate the performance posture. Despite this, the practice of the prolonged sound is too boring for children, who remain passive in this training and always muddle through the training. It leads to the unstable skills and problems in formal performance of children, thus destroying the performance rhythm of the entire band. Therefore, the school must pay more attention to the basic skill of prolonged sound training and supervise students to practice carefully. It should provide specialized practice places and time for children and ask students to think carefully and summarize the practice experience when practicing so as to improve playing skills.

\section{Importance of Rhythm and Speed Practice}

Any instrument will have a certain demand for the rhythm of the player; the sense rhythm is the most basic requirement for music-related personnel. If students do not have a very good sense of rhythm and speed, it will certainly have a certain impact on the performance of the overall performance and influence the performance effect. Schools should make an accurate provision for children's daily training. Firstly, require the children to grasp the speed of practice; they should also emphasize quality while pursuing speed. At the beginning of the practice, they must slow down the rhythm, and slowly master the feeling, to find their own defects of performance, and then correct the problems, and slowly cultivate a good sense of music. Second, students are required to be able to master the music, and also create an enough understanding of the composer's intention. But now the fact is that although most of the schools have certain requirements, yet the children still do not like this training; they like the rapid practice method, for they hold that practice can make them quickly grasp the music and save time. But in this practice mode, slowly there will be drawbacks. In the formal performance, it is very easy to have performance mistakes. The children must have enough understanding of the purpose of slow practice; the slow practice is to be perfect in the official performance. In addition, the children should also have enough exercise on the strength of the experience and should gradually grasp the rhythm of strength in the speed of practice. Finally, we must emphasize that the rhythm of all bands is the most important part of the performance and is the basis of all music.

\section{Necessity of Listening Feeling Practice}

All the beautiful songs are appreciated through the ears. Therefore, in the practice of trumpet, be sure to listen carefully with the ears and the ear is the best tonic teacher. In the usual training, carefully use their own ears to find the problems and record all the dissatisfying places, flaws and 
even the comfortable places so as to sum up experience. Through ears, players can quickly find some performance defects. Firstly, they can judge the intonation. All the performances must rely on ears, rather than fingers and breaths. Players can correct the intonation problems in the performance process and also judge the tone. The tone played by the trumpet is the most important; it directly affects the effect of trumpet playing. Players can use the ears to identify out in time. It is very important for the children to practice listening training. The school must be provided for this. First of all, ask the professors to teach the children to practice both listening and the basic training; in the usual basic training, slowly accumulate experience and master the ear;s basic skills of grasping the sound, and pitch. Second, it is necessary to ask the children to have enough sensitivity in the official performance of the sound, and promptly correct the possible tone or pitch problems. In the usual daily training, continuously strengthen it and keep applying ears to the training process and master the good practice habits. The application of the ear in the process of playing can quickly improve the children's playing skills and improve the completion of the entire band.

\section{Understanding Practice of Music}

In daily practice, if children do not have any ideas, the repeated mechanical training every day is very boring, which is easy to kill a person's will and easy to produce resistance. So the teachers must ask the children to understand the songs to be performed and make children understand and experience the emotions that the original composers want to express, then walk into music and be integrated into music. Everyone has a different understanding of music, and even everyone has a different understanding and sentiment of the same music in different stages of life. The children should be able to have their own feelings about the music and integrate their own understanding into them so as to play carefully. Immerse themselves into the music and the daily basic training will not be very irritable and mastery of the skills will be more simple. The children must know and understand that every note is vital and they have their own ideas and emotions. In the training, children should be able to communicate with these notes and perfectly show the emotions.

\section{Practice of Team Ensemble}

As the basic skills of the children began to have some achievements, we should begin to consider the cooperation with the team. But the premise of the team ensemble is that the children can join the team under the premise that the solo performance abilities of children are strong enough. The following requirements of entering the team should be met, such as mastery of the correct way of breath, playing posture, mastery of the sound by ears and so on. Musical instruments can play a magnificent melody through mutual cooperation. So the training of team ensemble is very necessary. The requirements of the team performance for each person are very high. It also has great requirements for the understanding of them, which should be slowly cultivated in the long-term mutual cooperation. Once it is formed, success is easy to attain.

The band refers to a complex whole that is combined by a variety of instruments. The band's performance requires the mutual cooperation of the instrument elements. There is a great demand for the performance of each instrument. Colleges and universities need to perfectly infuse the concept of each student's heart. It is a very important teaching task to strengthen the awareness of cooperation among children. The band's performance is very complex and huge, which cannot be perfect combination at the very beginning and requires the slow practice and training. And the overall cooperation cannot be done in the beginning, which should be separated into several groups, starting from small training to the entire training so as to reach the final entire cooperation. In this practice, the children can not only play the ensemble of the single part of the band and also work together in the overall ensemble. The benefits of this training model are diversified, which can cultivate a variety of cooperation ways, so as to enrich the content and cultivate a sense of 
cooperation.

At present, students' band groups exist in both the music colleges and common higher institutions, which not only represent the hope of a school to music, but also symbolize the humanistic spirits of the universities and embodies the entire quality of universities. Student bands are also the bridges of the exchanges and communications of the universities, which have made great contributions to the communications and exchanges of the universities. All in all, students must stick to it perseveringly in daily learning of trumpet. One day, the children will be able to master the skills of playing and the band's performance will be well done.

\section{Summary}

Music academies must attach more importance to the trumpet teaching; trumpet is an essential part of the band performance. To perfectly integrate the tones of the trumpet to the performances cannot do without the accumulative practice for multiple years. In daily teaching of classroom, teachers must cultivate children's ability to understand music and listen to the ears. Only when these factors are available can students slowly have the basic ability of trumpet musicians. To be unique in the trumpet performance requires more experience and talent. Hope that the colleges and universities can conduct more thinking of the teaching methods of the trumpet, strive to find more suitable learning modes and cultivate more professional talents for the future of the motherland.

\section{References:}

[1] Zhang Xinlin. Cultivation of Best Mental State of Trumpet Performance[J]. Beijing: Journal of PLA Art College, 2010.

[2] Shang Lijun. Exploration of Natural and Physiological Characteristics of Trumpet[M]. Shanghai: Shanghai Foreign Language Education Press, 2012.

[3] Wang Jianxin. Role of Trumpet in Different Bands and Artistic Expression. Journal of PLA Foreign Languages Institute, 2014, (1) 